\title{
Neurosurgical management of brain and spine tumors in the COVID-19 era: an institutional experience from the epicenter of the pandemic
}

\author{
Remi A. Kessler ${ }^{1}$. Jeffrey Zimering ${ }^{1} \cdot$ Jeffrey Gilligan ${ }^{1} \cdot$ Robert Rothrock $^{1} \cdot \operatorname{lan}$ McNeill $^{1} \cdot$ Raj K. Shrivastava $^{1}$. \\ John Caridi ${ }^{1}$. Joshua Bederson ${ }^{1}$. Constantinos G. Hadjipanayis ${ }^{1}$ (i)
}

Received: 17 April 2020 / Accepted: 27 April 2020 / Published online: 5 May 2020

(c) Springer Science+Business Media, LLC, part of Springer Nature 2020

\begin{abstract}
The challenges of neurosurgical patient management and surgical decision-making during the 2019-2020 COVID-19 worldwide pandemic are immense and never-before-seen in our generation of neurosurgeons. In this case-based formatted report, we present the Mount Sinai Hospital (New York, NY) Department of Neurosurgery institutional experience in the epicenter of the pandemic and the guiding principles for our current management of intracranial, skull base, and spine tumors. The detailed explanations of our surgical reasoning for each tumor case is tailored to assist neurosurgeons across the United States as they face these complex operative decisions put forth by the realities of the pandemic.
\end{abstract}

Keywords Neurosurgery $\cdot$ Tumor $\cdot$ Intracranial $\cdot$ Skull base $\cdot$ Spine $\cdot$ Resection $\cdot$ Pandemic $\cdot$ Virus

\section{Introduction}

The severe acute respiratory syndrome coronavirus 2 (SARS-CoV-2) and its associated coronavirus disease (COVID-19), is a new disease that manifested in late 2019 that was declared a global pandemic by the World Health Organization (WHO) on March 11, 2020 [1]. This virus originated in the city of Wuhan, China and then disseminated rapidly to over 100 countries. According to the Centers for Disease Control (CDC) surveillance system, as of April 13,2020 , there were 554,849 cases and 21,942 deaths in the United States [2]. The cases are now most highly centralized in the states of New York and New Jersey, with other significant clusters in Washington, Louisiana, California, and Michigan. At this present juncture, all 50 states have confirmed COVID-19 cases.

New York City (NYC) specifically provides some unique challenges to combatting COVID-19 due to the high-density population of the city and its outer boroughs. There are currently almost 190,000 COVID-19 cases in New York and more than 10,000 patients have died due to the coronavirus

Remi A. Kessler

remi.kessler@icahn.mssm.edu

1 Department of Neurosurgery, Icahn School of Medicine at Mount Sinai, The Mount Sinai Hospital, 1468 Madison Ave., New York, NY, USA at present time [3]. There are seven hospitals within the Mount Sinai Health System in various locations across the neighborhoods of Manhattan, Brooklyn, and Queens. The main teaching hospital is a 1141-bed, tertiary care academic center located on the Upper East Side of Manhattan. In addition to the aforementioned hospitals, the Department of Neurosurgery also provides coverage for the Elmhurst Hospital, a 545-bed Level I Trauma county facility in Queens, NY. This region of NYC has been particularly hit hard by COVID-19 due to the nature of the public hospital system and the dense patient population it serves.

COVID-19 has created unprecedented changes to healthcare delivery in NYC. Due to the staggering number of COVID-19 patients, the Mount Sinai Health System has reallocated resources and space for patients who become oxygen dependent and may ultimately require mechanical ventilation. Redeployment of attending neurosurgeons and residents to assist in covering a COVID-19 intensive care unit (ICU) has become essential in the fight against the coronavirus. All outpatient clinic visits have been transformed to telehealth visits and all resident teaching conferences are virtual.

The cancellation of all elective neurosurgeries was decided early in the NYC pandemic due to the diversion of regular post-operative beds into COVID-19 units, the re-direction of anesthesia and operating room (OR) staff to other units, the repurposing of OR anesthesia equipment 
for mechanical ventilation, the need for preservation of personal protective equipment (PPE), the closing of neurorehabilitation, and most importantly the protection of fresh postoperative patients from COVID-19. The regular OR capabilities have essentially ceased with the exception of emergency cases that require special approval by hospital leadership to proceed. As the pandemic marches forward into an unclear future, the definitions of "emergent" and "elective" procedures are not always so straightforward and as neurosurgeons, when to operate and when to delay becomes a complex decision-making process. With that said, a small subset of neurosurgical procedures is still taking place, specifically those that our surgeons have deemed necessary to operate on without delay to avoid impending death or irreversible harm to the patient. This is especially true with respect to brain and spine tumor cases in our neurosurgical oncology practice. Operative intervention is often required to relieve tumor mass effect on the brain and spinal cord in order to preserve and/or improve neurologic function. These procedures also permit proper diagnosis of malignancies that require urgent adjuvant therapies for ultimate improved survival of patients. However, a balance of when to use precious OR resources as well as the serious exposure risk to anesthesiologists, neurosurgeons, and OR staff during the intubation process, must be taken into consideration since all patients are presumed to be infected by the coronavirus. Certain patients who normally would undergo a resection may indeed require a biopsy since the disposition of the patients to rehabilitation is lacking. Other elderly patients with multiple comorbidities who are at increased risk for COVID-19 may not be proper candidates for any surgery. Furthermore, how to manage the surge in the backlog of cases that will require more urgent intervention at the conclusion of the pandemic is an unanswered question at this time. Despite all of the above circumstances, it is important that neurosurgical patients, who often have serious life-threatening conditions, are not neglected by our specialty even during a time of exceptional stress.

In this paper, we present our institutional neurosurgical oncology patient management approach for the treatment of brain and spine tumors during the COVID-19 pandemic in NYC. For each tumor type (cranial, skull base, and spine), we provide one case example that was managed differently from the perceived standard of care due to the COVID-19 crisis. The following example cases are solely to provide our guiding principles and experience at this unprecedented time in neurosurgical practice at a large tertiary care academic medical center in NYC, the heart of the coronavirus pandemic in the United States. Individual patient management should always remain at the discretion of the treating neurosurgeon's clinical judgement and expertise.

\section{Cranial tumor}

\section{Case 1}

The patient is a 75 year-old female who presented with fever, chills, and shortness of breath. The patient had a past medical history significant for multiple comorbidities, including hypertension, diabetes mellitus, chronic kidney disease, peripheral vascular disease, and parathyroid carcinoma status post parathyroidectomy. A chest $\mathrm{x}$-ray (CXR) revealed possible atypical bilateral pneumonia consistent with COVID-19. The patient underwent coronavirus nasopharyngeal swab testing which was negative. However, due to the possible pneumonia and close to a $30 \%$ false negative coronavirus testing by nasopharyngeal swab, the patient was admitted for further observation. After admission to the COVID-19 hospital ward, evaluation of the patient revealed she was having word finding speech difficulties and right sided weakness. MR imaging revealed a large $(5.7 \times 3.9 \times 2.9 \mathrm{~cm})$, heterogenous contrast-enhancing left frontoparietal mass causing cerebral compression, edema, and midline shift of $7 \mathrm{~mm}$ (Fig. 1). The patient was started on high-dose steroids and given antiepileptics. The family was counseled on the need for a tissue diagnosis and possible surgical decompression of the tumor since the patient had evidence of right sided weakness from the tumor mass effect. However, after discussion with the family and the medical team, the decision was made to just perform a stereotactic biopsy of the mass for diagnostic purposes. Since there was no possibility for any postoperative rehabilitation due to closing of the rehabilitation unit, and the high likelihood the patient had a high-grade glioma in the setting of advanced age and comorbidities, a craniotomy was not offered to the patient. The stereotactic biopsy was performed and revealed a glioblastoma tumor. She also also had a CT scan of the chest which showed improvement of the findings on her initial CXR. The patient is starting radiation therapy and temozolomide at the end of month of April 2020 and will require high dose steroids due to edema and mass effect of the tumor.

\section{Skull base tumor}

\section{Case 2}

The patient is a 71 year-old female with a past medical history of hypertension and blindness secondary to glaucoma who presented to an outside hospital with right-sided facial palsy, altered mental status, and a fall eight days prior with no loss of consciousness or trauma. A head CT scan showed a large $(3.9 \times 3.4 \times 5.5 \mathrm{~cm})$ mass within the sella and suprasellar cistern with sellar expansion, moderate mass effect, 
Fig. 1 Cranial Tumor CasePreoperative MR Imaging
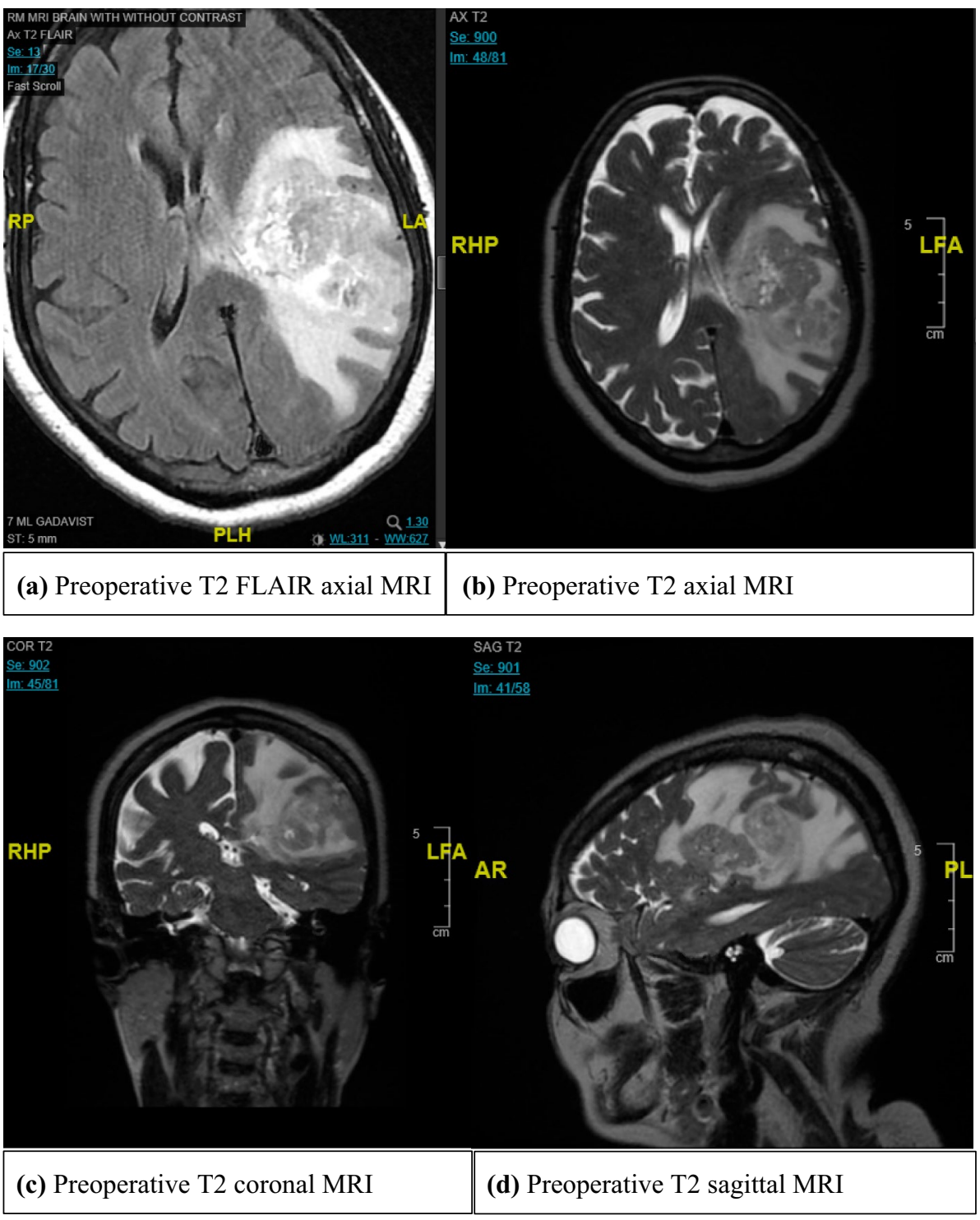

and some obstructive hydrocephalus. The patient was then emergently transferred to our hospital for surgical evaluation and possible CSF diversion. She then underwent MRI, which suggested a pituitary adenoma with significant displacement of the optic apparatus, cavernous sinus, third ventricle and midbrain, along with hydrocephalus (Fig. 2). She also underwent standard pituitary tumor lab work-up, with results showing normal levels of prolactin (17.2), AM cortisol (18.9), and TSH (1.049) and low levels of IGF-1 (27), free T4 (0.68), and ACTH (2.5). Given that the prolactin level was within normal limits, we did not elect to manage the tumor medically, since it was not a prolactinoma. The hydrocephalus was from the obstruction of the 3rd ventricle due to mass effect from the large pituitary tumor. The patient was brought to the OR and underwent a right frontal ventriculoperitoneal shunt (VP) shunt and endoscopic septal fenestration with a CERTAS programmable valve. The standard of care, an endoscopic transnasal transsphenoidal approach for maximal resection of tumor, was not offered at this time to the patient due to the COVID-19 crisis and the need to mitigate surgical exploration involving the nasopharynx as a nidus of infection. Instead, the patient was offered and underwent VP shunt placement to alleviate the emergent hydrocephalus. The patient did well postoperatively and was discharged to home on postoperative day two.

\section{Spinal tumor}

\section{Case 3}

The patient is a 63 year-old male with multiple myeloma who presented initially with neck pain and was found to have a pathologic fracture at $\mathrm{C} 2$. The overall alignment of the spine was maintained and there was no evidence of spinal 
Fig. 2 Skull Base Tumor Case-Preoperative MR Imaging

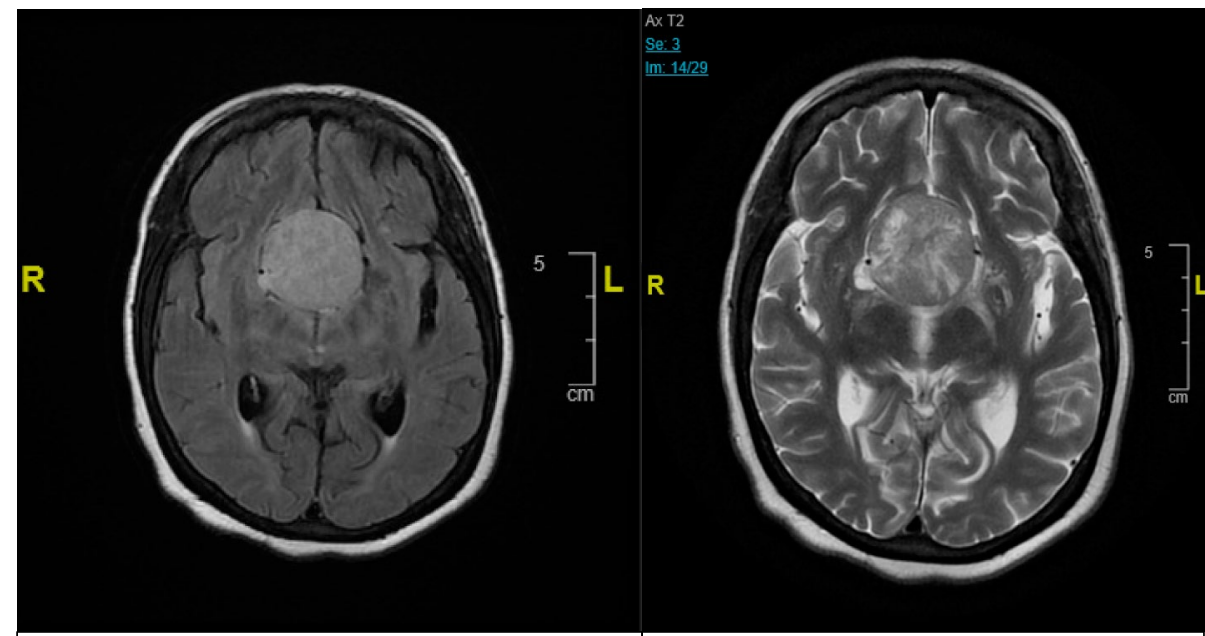

(a) Preoperative T2 FLAIR axial MRI

(b) Preoperative T2 axial MRI

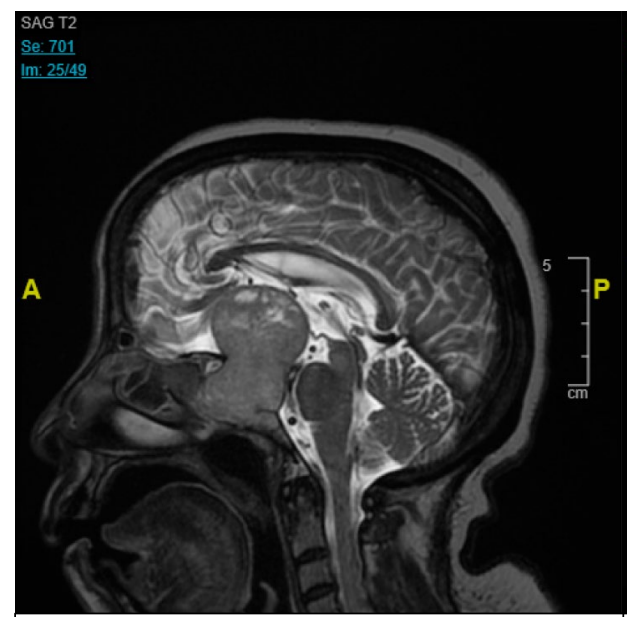

(c) Preoperative T2 sagittal MRI

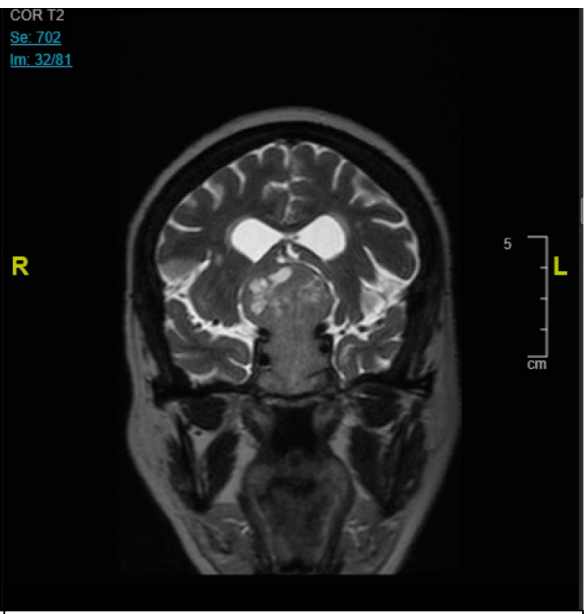

(d) Preoperative T2 coronal MRI cord compression. Given the lack of neurologic compromise and overall preserved alignment of the spine, it was decided that a minimally invasive approach could be attempted by neuro-interventional radiology. The patient underwent a $\mathrm{C} 2$ cement augmentation. Post procedurally, the patient did very well. However, he re-presented to the emergency department (ED) with worsening neck pain, bilateral hand numbness, and pneumonia. A cervical spine CT scan showed new dissociation between the odontoid and the cemented $\mathrm{C} 2$ vertebral body with resulting severe stenosis and spinal cord compression (Fig. 3). Given the underlying pneumonia and stable neurologic condition, the decision was made to keep the patient on bed rest in a rigid orthosis while he underwent treatment for the pneumonia and COVID-19 testing. Once the pulmonary issues resolved and the patient had multiple negative COVID tests, he was taken to the OR for a posterior decompression and fusion from C1-3. After surgery, his pain was significantly improved and his upper extremity numbness resolved. The initial recommendation was for inpatient rehab, however after prolonged discussion with the patient and his family, it was felt that he would be safer to be discharged home with home services and family education.

\section{Discussion}

The severity of the COVID-19 pandemic in the State of New York, and in particular New York City, has required neurosurgeons to dramatically alter their practice in a very short period of time. The diversion of essentially all hospital facilities and resources to combatting this highly contagious disease and the cancellation of all elective procedures has 


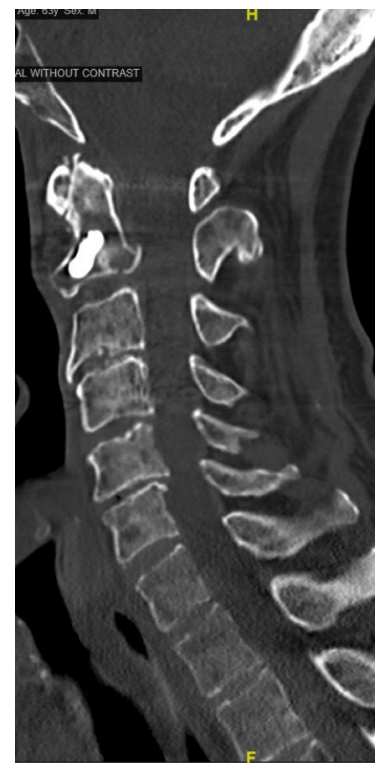

(a) Preoperative sagittal CT

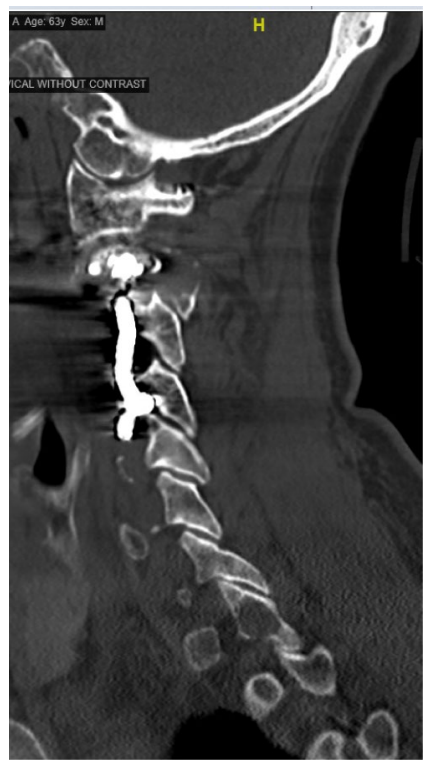

(b) Preoperative sagittal CT

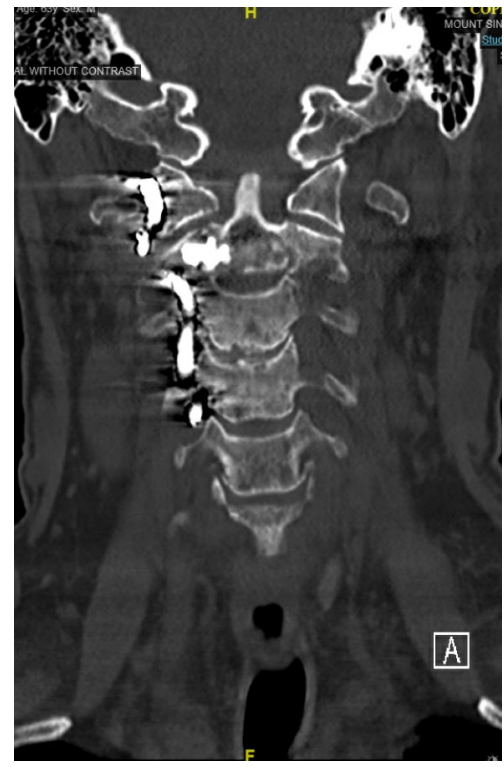

(c) Preoperative coronal CT

Fig. 3 Spine Tumor Case-Preoperative Cervical Spine CT Imaging

required a striking shift in patient management and surgical decision-making. COVID-19 provides difficulties in the treatment of all patients who necessitate hospital-level care - both medical and surgical - however it poses unique challenges in the management of neurosurgical tumor patients. This is due to several reasons, one of which is that many tumor patients tend to be of middle or older age, and the death rate for COVID-19 is significantly higher in older adults and for those with various cardiopulmonary comorbidities $[4,5]$. Patients with diabetes mellitus are also at much higher risk for severe complications [6, 7]. Second, patients with malignant brain or spine tumors may have undergone chemotherapy prior to surgical resection or in between subsequent resections, thus placing them in an immunocompromised state and further increasing their risk of acquiring COVID-19 [8]. Patients who have metastatic disease are also often immunosuppressed on systemic chemotherapeutic agents. Due to the highly contagious nature of COVID-19, it has even been recommended that cancer patients avoid treatment regimens that cause immunosuppression or have their dosages reduced should they become infected [9]. These are clearly dramatic changes from normal clinical practice, which reflect the severity of the COVID-19 virus. Risks of acquisition are only further exacerbated when a significant surgical procedure occurs that then necessitates a prolonged hospital stay. Taking the aforementioned aspects into consideration changes the calculus of the preoperative risk assessment but represents only one component of the surgical decision-making thought process needed in light of COVID-19.

For the cranial tumor surgeon, several factors come into play in deciding if and when to operate. In case \#1, the patient was originally admitted to the hospital for pneumonia-like symptoms, suspicious for COVID-19. She later experienced a variety of neurological symptoms, including focal deficits, completely unrelated to any prior medical history or her infectious workup. She was incidentally found to have a very large mass lesion with compression of surrounding structures, the likely cause of her new symptoms, and started on high-dose steroids. At Mount Sinai in the COVID-19 era, there have been no changes to the use or dosing of steroids to treat edema from brain tumors. Of note, steroids are even being used for patients with COVID-19 who experience acute respiratory distress syndrome (ARDS) [10]. Under non-COVID-19 circumstances, the routine care at our institution would have been maximal surgical resection with preservation of neurological function given that her lesion was in a surgically-amenable location. However, neurorehabilitation services are presently not available due to the closing of the normal rehabilitation unit and the rerouting of all its staff to COVID-19 units. As such, the decision to solely conduct a stereotactic biopsy for histopathologic analysis represents a deviation from our typical standard of care. While entirely out of the neurosurgeon's control, this is a serious issue for patients with malignant lesions, which are obviously time-sensitive for growth, in that these lesions 
can no longer be resected in a timely manner as there is now no postoperative care for them. The reasoning for the stereotactic biopsy in this case was that at the very minimum, there would be a definite diagnosis for this severely symptomatic patient, which could elucidate whether the tumor had malignant features. Additionally, a clear tissue diagnosis, despite the present incapability for maximal resection, would guide further treatment, including possible radiation therapy and chemotherapy, and patient expectations about next steps. This change from not offering maximal surgical resection for a tumor with clear effects of edema and compression is a remarkable departure from our traditional practice. Our decision-making shares similarities with the neuro-oncologic community consensus at this time for the treatment of gliomas, in that diagnostic surgery and adjuvant therapy can be delayed if the patient is stable and if further tumor expansion will not hinder complete resection later on [11].

In case \#2, the patient presented with a pituitary macroadenoma with altered mental status and falls due to mass effect from the large sellar lesion and associated obstructive hydrocephalus. The decision to solely place a VP shunt and withhold surgical resection represents a striking departure from our institutional neurosurgical standard of care for a symptomatic pituitary tumor. Treating the hydrocephalus expeditiously is in keeping with the general guidance recently put forth by the American Association of Neurological Surgeons/Congress of Neurological Surgeons (AANS/ CNS) tumor section and Society for Neuro-Oncology [12].

The endoscopic transnasal transsphenoidal approach for anterior skull base resection is cause for concern for the operating skull base neurosurgeon due to additional risk of the nasopharynx harboring the COVID-19 virus. Specifically, this is due to the high nasal viral titers and the potential for aerosol production during endonasal instrumentation. One particular study simulated surgical aerosolization during non-powered instrumentation, suction microdebrider, and high-speed drilling in cadavers after nasal fluorescein application [13]. They found that an intact surgical mask and VENT mask for endoscopy removed all detectable spread of aerosol particles. In addition, microdebrider and
Fig. 4 Checklist for Booking Neurosurgical Cases during the COVID-19 Outbreak, from the UCSF Department of Neurosurgery. Reprinted with permission

\section{Checklist for Neurosurgical Cases During COVID-19 Outbreak}

\section{Determine level of urgency of case:}

$\square$ Assess for neurosurgical emergency (0-48 hours to OR). Emergent cases include:

$\square$ cranial trauma/infection.... TBI, depressed skull fractures, space occupying lesions, empyema/abscess $\square$ cranial tumor ................... pituitary apoplexy, tumor with mass effect

$\square$ cranial vascular ............... intracranial hemorrhage from ruptured aneurysm, AVM, and/or dAVF

$\square$ cranial CSF diversion ....... shunt obstruction, acute hydrocephalus

$\square$ functional ....................... hardware infections, sudden DBS battery failures

$\square$ spine .............................. spinal instability or spinal cord compression from fracture, tumor, or infection

$\square$ spine, disc disease ........... cauda equina, nerve root compression with progressive motor deficit

$\square$ In addition, any case meeting the following criteria:

$\square$ acute and progressive neurological symptoms referable to focal lesion on imaging, AND

$\square$ determined to be an emergency by board certified neurosurgeon

$\square$ Assess for neurosurgical urgency (2-14 days to OR). Urgent cases include:

$\square$ any case requiring surgery within a 14 day period that does not meet above criteria

$\square$ Assess for purely elective cases

$\square$ any case not meeting criteria for urgent or emergent cases (defined above)

\section{Determine availability of operating room:}

$\square$ Blood is available in blood bank? If not, abort surgical scheduling

$\square$ Sufficient PPE (per infection control protocol) available? If not, abort surgical scheduling

$\square$ Assess viral surge level in the local community

If green, $\square$ schedule elective and urgent cases, proceed with emergent cases

If yellow, $\square$ schedule urgent cases, proceed with emergent cases

If red, $\square$ schedule urgent cases per multi-disciplinary periop committee (surgeons + anesthesia), proceed with emergent cases

If black, $\square$ proceed only with emergent cases only

Determine availability of post-operative beds:

$\square$ Assess need for ICU post-operatively

$\square$ Determine possibility of floor placement after surgery

$\square$ Check with nursing at designated post-operative destination for comfort receiving patient

Surgery was able to be scheduled with available post-operative bed secured?

$\square$ Yes, book surgery

$\square$ No

$\square$ Repeat checklist for next 21 days to ensure no changes in status

$\square$ If still unable to book surgery, explain to patient the need to cancel surgery, and re-schedule after COVID-19 outbreak 
cold instrumentation use did not produce detectable aerosols, however high-speed drill generated remarkable aerosol spread, with significant risk to the endoscopic skull base surgeon.

Preliminary safety recommendations for otolaryngologic surgery based on anecdotal experience include the complete postponement of endoscopic nasal procedures in patients with COVID-19 or those who cannot be tested [14]. One other group recently described precautions to be taken in endoscopic endonasal surgery, but noted that they are based only on personal communication with international colleagues in China, Italy, Iran, the United Kingdom, and Greece reporting on their individual experiences. In summary, they describe a number of physicians becoming infected with COVID-19 as a result of endoscopic procedures, with some resulting in deaths, and emphasize to only perform urgent/emergent surgery [15]. In the case when surgery is critical, a number of specific precautions with respect to intubation and extubation procedures have also been recommended [16].

In case \#3, the patient's re-presentation to the ED with severe cervical stenosis and spinal cord compression after a neuro-interventional radiology procedure for a vertebral compression fracture was complicated by additional newonset pneumonia. Given the high likelihood of a COVID-19 etiology of the pneumonia and the fact that the patient was mostly neurologically intact, the decision was made for the patient to be treated for the infection first, while being maintained in rigid orthosis in bed, and await surgery until the pneumonia had cleared. In non-COVID-19 times, patients with pneumonia are generally not operated on unless it is a surgical emergency. That is consistent with the management plan here. However, given the highly contagious nature of COVID-19 and its potential complications, extra precautions were taken to ensure the pneumonia was entirely cleared prior to bringing the patient to the OR. In other words, the preference was for the patient to be maintained in rigid orthosis as long as necessary, which is a change from standard of care.

One other institution, the University of California at San Francisco, has described their own protocols for the scheduling of neurosurgical cases and for neurosurgical coverage throughout the COVID-19 pandemic [17]. Of particular interest to the topic of this paper is the checklist they developed to determine what constitutes an emergent versus urgent versus elective neurosurgical procedure (Fig. 4). Our surgical decision-making process for tumor cases in light of COVID-19 is overall consistent with their global approach. Nevertheless, our institutional experience and subsequent changes to patient management will inevitably differ given that our hospital is located in the epicenter of the pandemic across the US, and faces distinct challenges, including an intensive care unit that is currently at triple its normal full

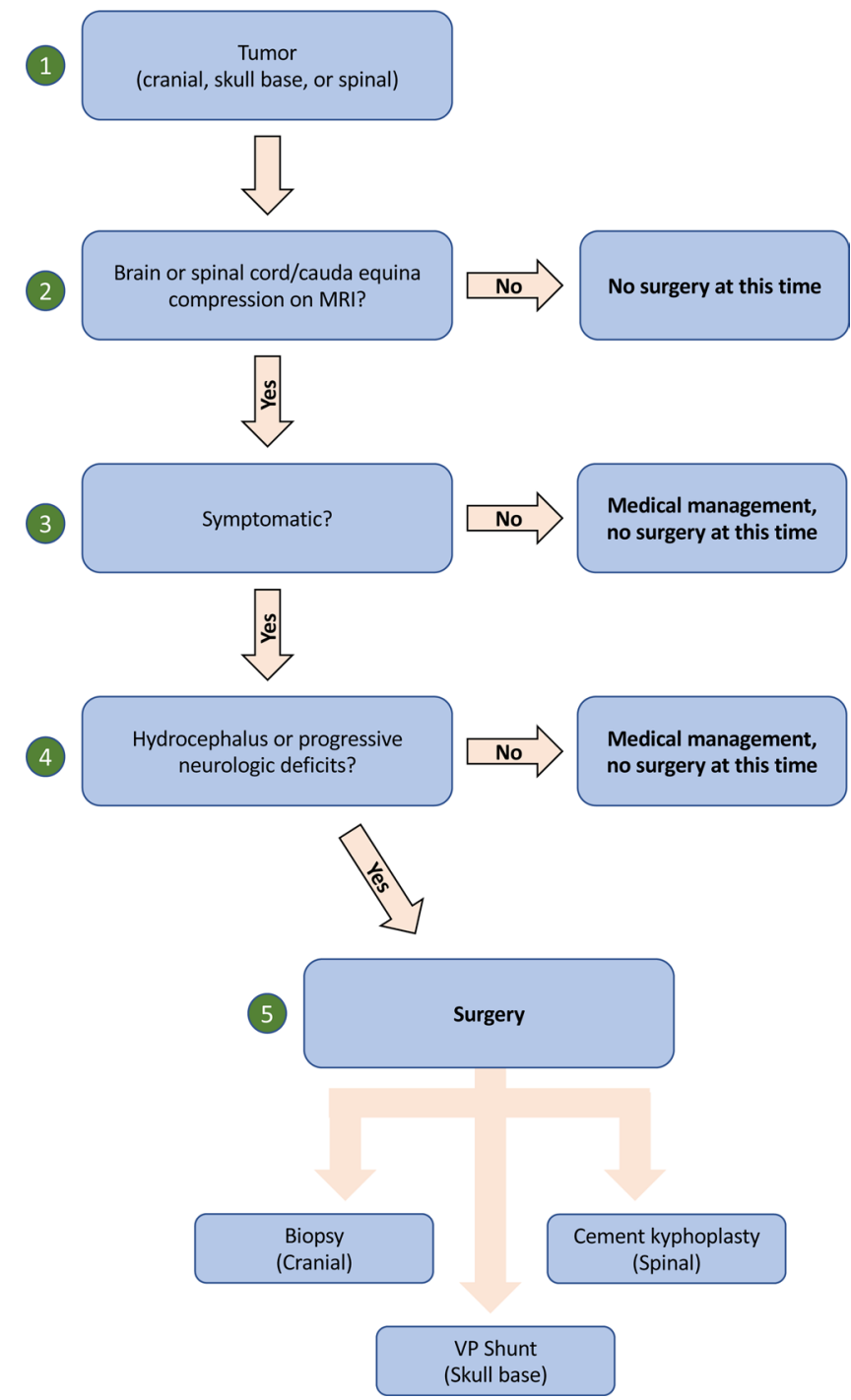

Fig. 5 The Mount Sinai Department of Neurosurgery Tumor Management Algorithm

capacity due to COVID-19 patients. To address these concerns, we have developed our own institutional algorithm for the management of brain and spinal tumors (Fig. 5), which is put to use in all three cases outlined above.

Despite all the above tumor-specific considerations, protecting our surgeons, residents, nurses and all other medical staff must be of highest priority. Transmission of the virus necessarily increases in the close proximity of the hospital, placing both medical staff and patients at risk. Specifically, the intubation process is considered one of the highest risk procedures for viral droplet spread [18, 19]. As the vast majority of neurosurgical procedures are conducted under general anesthesia, minimizing cases to those that are only truly emergent also protects our anesthesiologists, neurosurgeons, residents, and OR staff from acquiring the virus. As was done in our institution's tumor 
cases described above, maximal OR precautions were taken at all times throughout the intubation process and in the post-operative setting. Patients were generally considered to be COVID-19 positive until proven otherwise, and multiple rounds of testing were sent as soon as possible, when available. Furthermore, not taking away from other needed hospital resources, including ICU beds with ventilators, nursing capabilities, and personal protective equipment (PPE), should be considered in circumstances in which delaying surgery causes no harm to the patient. It is important to note that different hospitals have varying levels of available resources, and given that our institution is a large tertiary-care academic hospital at the epicenter of the pandemic, our facilities may differ widely from those at community hospitals. Such differences necessarily impact patient management in a time of crisis.

\section{Conclusion}

The COVID-19 global pandemic and its colossal impact on all hospitals around the world has necessitated a vast change in standard neurosurgical oncology practice to accommodate for shifting resources. In this report, we provide examples of the Mount Sinai Hospital (New York, NY) neurosurgical experience and clinical reasoning for the changes in management of brain and spine tumors. We continue to operate on neurosurgical emergencies, evaluate tumors on a case-by-case basis, and make alterations or delay procedures when clinically feasible without causing any harm to the patient. While we would operate on all the cases above in a timely fashion under normal circumstances, the reality of the COVID-19 pandemic necessitates that neurosurgeons balance providing the best care for their patients while minimizing the spread of the virus.

Funding This study did not receive any funding.

\section{Compliance with ethical standards}

Conflicts of interest Remi A. Kessler, Jeffrey Zimering, Jeffrey Gilligan, Robert Rothrock, Ian McNeill, John Caridi, Joshua Bederson has no conflict of interest.

Constantinos G. Hadjipanayis is a medical advisor to NX Development Corp, is entitled to royalties from NX Development Corp, and is a consultant to Synaptive Medical (not related to the content of this study).

Ethical approval This article is a general institutional overview of department regulations that include three retrospective case reports. These patients' medical records were reviewed under a Mount Sinai IRB-approved protocol.
Informed consent Informed consent was not required in this study as they were solely case reports with all patient information completely de-identified.

Research involving human and animal rights All procedures performed in studies involving human participants were in accordance with the ethical standards of the institutional and/or national research committee and with the 1964 Helsinki declaration and its later amendments or comparable ethical standards.

\section{References}

1. WHO Director-General's Opening Remarks at the Media Briefing on COVID-19 - 11 March 2020. Web. https://www.who.int/ $\mathrm{dg} /$ speeches/detail/who-director-general-s-opening-remarks-atthe -media-briefing-on-covid-19---11-march-2020. Accessed April 12, 2020.

2. Coronavirus Disease (COVID-19). Cases in U.S. Centers for Disease Control. Web. https://www.cdc.gov/coronavirus/2019-ncov/ cases-updates/cases-in-us.html. Accessed April 14, 2020.

3. Coronavirus: New York region explores reopening as cases 'plateau.' BBC News. Web. https://www.bbc.com/news/world-uscanada-52274969. Accessed April 13, 2020.

4. Guan WJ, Liang WH, Zhao Y et al (2020) Comorbidity and its impact on 1590 patients with Covid- 19 in China: A Nationwide Analysis. Eur Respir J. https://doi.org/10.1183/13993003.00547 $-2020$

5. Yang J, Zheng Y, Gou X et al (2020) Prevalence of comorbidities in the novel Wuhan coronavirus (COVID-19) infection: a systematic review and meta-analysis. Int J Infect Dis. https://doi. org/10.1016/j.ijid.2020.03.017

6. Klonoff DC, Umpierrez GE (2020) COVID-19 in patients with diabetes: risk factors that increase morbidity. Metabolism. https ://doi.org/10.1016/j.metabol.2020.154224

7. Gupta R, Ghosh A, Singh AK, Misra A (2020) Clinical considerations for patients with diabetes in times of COVID-19 epidemic. Diabetes Metab Syndr. 14(3):211-212. https://doi.org/10.1016/j. dsx.2020.03.002

8. Al-shamsi HO, Alhazzani W, Alhuraiji A et al (2019) A practical approach to the management of cancer patients during the novel coronavirus disease, (covid-19) pandemic: an international collaborative group. Oncologist. https://doi.org/10.1634/theoncolog ist.2020-0213

9. Zhang L, Zhu F, Xie L et al (2020) Clinical characteristics of COVID-19-infected cancer patients: A retrospective case study in three hospitals within Wuhan. China Ann Oncol. https://doi. org/10.1016/j.annonc.2020.03.296

10. Poston JT, Patel BK, Davis AM (2020) Management of Critically Ill Adults With COVID- 19. JAMA. https://doi. org/10.1001/jama.2020.4914

11. Mohile NM, Blakeley OJ, Gatson TN, Hottinger A, Lassman $\mathrm{AB}$ et al (2020) Urgent Considerations for the Neuro-oncologic Treatment of Patients with Gliomas During the COVID-19 Pandemic. Neuro-Oncology. https://doi.org/10.1093/neuonc/noaa0 90

12. Ramakrishna R, Zadeh G, Sheehan JP, Aghi MK (2020) Inpatient and outpatient case prioritization for patients with neuro-oncologic disease amid the COVID-19 pandemic: general guidance for neuro-oncology practitioners from the AANS/CNS Tumor Section and Society for Neuro- Oncology. J Neurooncol. https://doi. org/10.1007/s11060-020-03488-7 
13. Workman AD, Welling DB, Carter BS et al (2020) Endonasal instrumentation and aerosolization risk in the era of COVID-19: simulation, literature review, and proposed mitigation strategies. Int Forum Allergy Rhinol. https://doi.org/10.1002/alr.22577

14. Givi B, Schiff BA, Chinn SB et al (2020) Safety Recommendations for Evaluation and Surgery of the Head and Neck During the COVID-19 Pandemic. JAMA Otolaryngol Head Neck Surg. https ://doi.org/10.1001/jamaoto.2020.0780

15. Patel ZM, Miranda-Fernandez J, Hwang PH, Nayak JV, Dodd $\mathrm{R}$ et al. PRECAUTIONS FOR ENDOSCOPIC TRANSNASAL SKULL BASE SURGERY DURING THE COVID-19 PANDEMIC. Web. https://med.uth.edu/orl/wp-content/uploads/sites /68/2020/03/Update-on-Precautions-Regarding-Endoscopic-Proce dures-and-COVID-19-2020-03-22.pdf. Accessed Aprl 15, 2020.

16. Vlessides M. Report From Wuhan: Hard-Won Insights From China. Infectious Disease Special Edition. 15 Apr 2020. Web.
https://www.idse.net/Covid-19/Article/04-20/Report-FromWuhan-Hard-Won-Insights-From-China/57998. Accessed April 15, 2020.

17. Burke JF, Chan AK, Mummaneni V et al (2019) Letter: the coronavirus disease, global pandemic: a neurosurgical treatment algorithm. Neurosurgery. https://doi.org/10.1093/neuros/nyaa116

18. Cheung JC, Ho LT, Cheng JV, Cham EYK, Lam KN (2020) Staff safety during emergency airway management for COVID-19 in Hong Kong. Lancet Respir Med. https://doi.org/10.1016/S2213 -2600(20)30084-9

19. Anesi GL. Coronavirus disease 2019 (COVID-19): Critical care issues. In: Manaker S, ed. UpToDate. Waltham, Mass.: UpToDate; 2014. www.uptodate.com. Accessed April 14, 2020.

Publisher's Note Springer Nature remains neutral with regard to jurisdictional claims in published maps and institutional affiliations. 